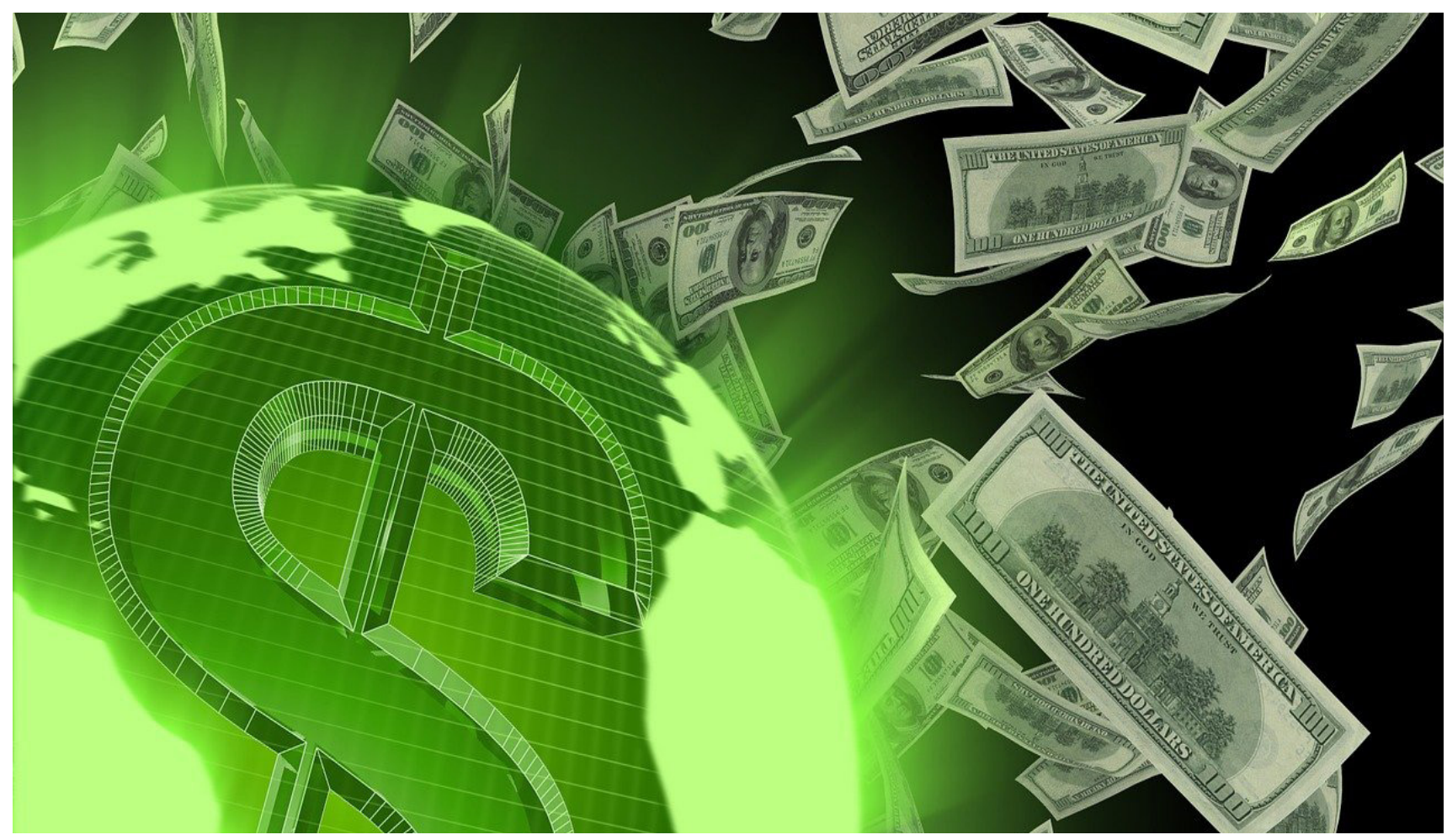

\title{
Estrategias para impulsar el mercadeo verde en las empresas de Cúcuta
}

\section{Strategies \\ to \\ promote \\ green marketing

Stiven David Rincón-Jaimes

Estudiante de Negocios Internacionales sd_rincon@fesc.edu.co,

Fundación de Estudios Superiores Comfanorte, Cúcuta, Colombia

Laura Viviana Machuca-García

Estudiante de Negocios Internacionales Iv_machuca@fesc.edu.co, Fundación de Estudios Superiores Comfanorte, Cúcuta, Colombia
Sory Samira Rondón-Castellanos

Estudiante de Negocios Internacionales, ss_rondon@fesc.edu.co

Fundación de Estudios Superiores Comfanorte, Cúcuta, Colombia 
Cómo citar: Rincón-Jaimes, S.D., Machuca-García, L.V. , Rondón-Castellanos, S.S. (2019). Estrategias para dar a conocer la importancia del mercadeo verde en las empresas de Cúcuta norte de Santander. Reflexiones contables (Cúcuta), 2(2), 62-68.

\section{Resumen}

El presente artículo de investigación tiene como objetivo Crear estrategias para dar a conocer la importancia del mercadeo verde en las empresas de Cúcuta norte de Santander. Los cambios sociales que se han venido dando en la actualidad, ha provocado cambios en los consumidores con respecto al consumo de los productos, por una parte la estructura de los valores de los individuos ha hecho consecuentemente que cambie las costumbres y hábitos de alimentación, y no solo eso, sino también los modos y rutinas relacionadas con la compra. En este sentido, han proliferado nuevos productos y servicios como los productos ecológicos, que actualmente están adquiriendo gran importancia debido a los cambios en la estructura social de valores, dando lugar a nuevas tendencias y patrones de consumo con un enfoque de cuidado al planeta.

Palabras clave: Estrategias, Planeta, Consumo, Mercadeo verde, Empresas.

\section{Abstract}

The present research article aims to create strategies to raise awareness of the importance of the green market in the companies of Cúcuta Norte de Santander. The social changes that have been taking place today have caused changes in the consumers with respect to the consumption of the products, on the one hand the structure of the values of the individuals has consequently made that change the habits and habits of feeding, and not only that, but also the modes and routines related to the purchase. In this sense, new products and services such as organic products have proliferated, which are now becoming very important due to changes in the social structure of values, giving rise to new trends and patterns of consumption with a care approach to the planet.

Keywords: Strategies, Planet, Consumption, Green marketing, Companies. 


\section{4}

\section{Introducción}

En la actualidad existen muchas empresas que están adoptando los términos de Responsabilidad Social, Sustentabilidad y Marketing Ecológico, como componentes básicos de la filosofía y gestión de la organización, por lo que está dando lugar a la reinvención de los productos y servicios.

Cuidando que, en la elaboración, comercialización y prestación de éstos, se tenga en cuenta que las necesidades a satisfacer de los consumidores deben ser acordes a los intereses presentes y futuros de la sociedad, conscientes del respeto al entorno natural, social y económico. Si bien lo que mueve al Marketing es el interés por incrementar las ventas y el consumo de un determinado producto, el Marketing Ecológico está orientado hacia un público medioambientalmente sensibilizado. Esta disciplina tiene repercusiones significativas positivas en la actitud de la empresa respecto al medio ambiente, $y$, a la vez, se convierte en un instrumento sensibilizador sobre el consumo responsable, por lo que la entidad, sociedad y medioambiente salen beneficiados.

Es por ello que en este trabajo se hace hincapié sobre la importancia y los beneficios que implica practicar el Marketing Ecológico como estrategia, y la necesidad de trabajar y mejorar puntos importantes de su responsabilidad social. Si bien, dar el primer paso es difícil, el empresario debe de ser consciente que hoy en día, la mirada está puesta en las empresas sustentables que se preocupan por mantener un equilibrio entre la sociedad, la economía y el medio amiente.

El Marketing Ecológico es una rama de la mercadotecnia social, en la que se busca adaptar los esfuerzos en esta área, para facilitarle a la organización un enfoque ambiental tanto social como empresarial. La idea de indagar sobre este tema, surge debido a que hoy en día debe existir una relación equilibrada entre la empresa y el medio ambiente. Las organizaciones juegan un papel protagonista en la búsqueda y aporte de soluciones tecnológicas a los problemas medioambientales, para la empresa el medio ambiente constituye un mercado en rápida expansión y una oportunidad de negocio y de creación de empleo, constituyendo además, una parte importante en la imagen, obtención y maximización de beneficios y una minimización de residuos peligrosos en el medio ambiente (Acosta, 1985).

El Marketing Ecológico se orienta a dos áreas: la social que va enfocada a los clientes y consumidores, y la empresarial, que se enfoca a la empresa internamente.

Respecto al área social hace referencia al conjunto de acciones llevadas a cabo por institucionessinfines delucro (administraciones, grupos ecologistas) para difundir ideas y comportamientos medioambientalmente deseables entre los ciudadanos y los distintos agentes sociales y económicos. Tiene como objetivos: informar / educar sobre temas medioambientales, estimular acciones beneficiosas para el medio ambiente, cambiar comportamientos nocivos para el entorno natural y modificar los valores de la sociedad en cuanto a la ecología. (Sanchez, 2000).

En cuanto al área empresarial, busca realizar esfuerzos internamente en la empresa para lograr ofertar productos y servicios que generen un menor daño al entorno, dichos esfuerzos van desde una nueva selección de materias primas, nuevas formas de transportar dichos productos, ahorro de recursos dentro de la empresa, y acciones en pro del ambiente, como son las reforestaciones o patrocinio para cuidado y preservación de aéreas verdes (parques, jardines), áreas ambientales protegidas (bosques, santuarios), o incluso de alguna especie animal (puma, ballena azul). 
Toda esta información genera más detalle e información de este mercadeo.

\section{Objetivo general}

- Crear estrategias para dar a conocer la importancia del mercado verde en las empresas de Cúcuta Norte de Santander.

\section{Objetivos específicos}

- Analizar la literatura referente al Marketing Ecológico, Responsabilidad Social Empresarial y Sustentabilidad, para fundamentar el marco teórico de la investigación

- Identificar los aspectos de esa práctica susceptibles de mejora, para fundamentar la propuesta.

- Fundamentar teórica y empíricamente una propuesta orientada a mejorar la importancia del mercado verde en las empresas de Cúcuta norte de Santander.

\section{Justificación}

El uso del Marketing Ecológico se halla estrechamente ligado al desarrollo sostenible, ya que equivale a optimizar tres objetivos: crecimiento económico, equidad social y valor ecológico. El desarrollo de productos o servicios respetuosos con el medio ambiente es la clave para reducir el impacto negativo en él. Es decir, se pueden realizar diversas acciones para disminuir el impacto ambiental de las empresas y una de ellas es el uso del Marketing Ecológico.

El beneficio social y económico que se deriva de este estudio es orientar la importancia del mercado verde para mejorar y reducir futuros costos en la creación, manejo o distribución del producto o servicio. Asimismo, este trabajo se realiza con el propósito de que su contenido sirva tanto a estudiantes y académicos interesados para profundizar sus estudios sobre el tema, así como expandir el conocimiento del mismo.

Desde el punto de vista de la revolución industrial la actividad humana solo se ha centrado en el crecimiento económico de las naciones y en el sustento del nivel de vida de las personas. Cabe hacer hincapié en la palabra bienestar, que se asocia a la idea de alcanzar cada vez mayores niveles de satisfacción y comodidad, esto repercute en grandes producciones por lo tanto, diversos sistemas de abastecimiento, comunicaciones, transporte etc. Así cuanto más se produce y se consume el nivel de satisfacción es mejor. El problema radica en cuanto más se produce se necesita mayores recursos y cuanto más se consume mayores desperdicios se generan (Landazal, 2009).

\section{Marco teórico}

En la actualidad la escasa educación ambiental, la falta de información precisa, la ausencia de reglas con falta de rigor y la escasa cultura con respecto al cuidado del medio ambiente en Colombia hace que este tema toque fondo en la conciencia social. Por consiguiente, a estos problemas se les ve por consecuencia el calentamiento global, la contaminación de aire, suelo y ríos, la tala de árboles, pero ante todo lo inquietante de esto es la despreocupación y falta de compromiso de los habitantes con el medio ambiente aunque sea un problema que nos afecta a todos. La preocupación por el medio ambiente ha llegado a ser parte de la vida de algunas personas alrededor del mundo, y esto ha generado un compromiso por parte de las marcas en la creación de estrategias comunicativas que motiven a la sociedad a tener una perspectiva más ecológica de la vida.

El marketing ecológico es una forma de percibir y llevar a cabo la relación de 


\section{6}

intercambio, con el fin de que sean satisfactorias para las partes que intervienen, la sociedad y el entorno natural, mediante el desarrollo, valoración, distribución y promoción por una de las partes de los bienes, servicios o ideas que la otra parte necesita, de forma que, ayudando a la conservación del medio ambiente, estos contribuyan al desarrollo sustentable de la economía y la sociedad. (Hartmann, 2004).

Según (Terrón, 2007) el marketing ecológico es el resultado de complementar el factor medioambiental en las funciones de marketing y por lo tanto, la aplicación de un marketing operativo que reafirme al consumidor el producto que por su naturaleza, presentación, costo e impacto medioambiental, responda mejor a sus necesidades. El marketing ecológico nace del marketing social, situándolo como intermediario entre los intereses individuales y el interés de los consumidores.

Según (Chamorro, 2001) desde una perspectiva social el marketing ecológico es parte del marketing social ya que son actividades donde el principal objetivo es estimular y facilitar la aprobación de ideas o comportamientos sociales que se consideran provechosos para la sociedad, por medio de tratar de frenas o desincentivar aquellas otras ideas o comportamientos que se juzgan perjudiciales.

"Por otra parte, motivadas tanto por la evolución de la concienciación de los consumidores y de los dirigentes empresariales como por una legislación medioambiental cada vez más estricta, las empresas han respondido a las nuevas exigencias de sus clientes, incorporando programas y políticas de protección del medio ambiente en su sistema de objetivos" (Catarina, 2008).

De acuerdo con (Chamorro, 2001) el marketing ecológico es el marketing que ponen en práctica las empresas que adoptan una orientación de marketing social para comercializar productos ecológicos, es decir, aquellas empresas que pretenden satisfacer las necesidades sociales y al mismo tiempo a las necesidades presentes de los clientes. En este contexto se define como un proceso de planificación, implantación y control de una política de producto, precio, promoción y distribución que permita conseguir los criterios a alcanzar por el marketing mix.

La inquietud por el deterioro ambiental no solo es una difícil tendencia social, es un fenómeno de marketing que está propiciando la aparición de un nuevo segmento de consumidores: los consumidores verdes. Estos consumidores no están solo preocupados por sus necesidades actuales sino también se encuentran preocupados por la naturaleza, esto fuerza de cierta manera a las organizaciones hacia una nueva manera de entender el marketing. Se puede definir el consumidor ecológico como aquel que expresa su preocupación por el medio que nos rodea en su conducta de compra, buscando productos que sean amigables con el medio ambiente. (Chamorro, 2001).

\section{Materiales y métodos}

Se establece que un diseño no experimental es: "La que se realiza sin manipular deliberadamente variables. Es decir, se trata de investigación donde no se modifican intencionadamente las variables independientes. Lo que se hizo en la investigación no experimental, fue observar fenómenos y como se dan en su contexto natural, para después analizarlos." (Sampieri, 2014).

La investigación no experimental es la búsqueda empírica y sistemática en la que el científico no posee control directo de las variables independientes, debido a que sus manifestaciones ya han ocurrido o a que son inherentemente no manipulados. Se hacen inferencias sobre las relaciones entre las 
variables, sin intervención directa sobre la variación simultánea de las variables independiente y dependiente (Kerlinger, 2002).

\begin{tabular}{|c|c|c|}
\hline FUENTE & CONTENIDO & ANALISIS \\
\hline $\begin{array}{l}\text { (Dinero, 2017) El } \\
\text { negocio del futuro }\end{array}$ & $\begin{array}{l}\text { Colombia es uno de los cinco paises de mayor diversidad } \\
\text { ecológica en el mundo, alberga el } 15 \% \text { de todas las especies } \\
\text { terrestres conocidas y es el segundo país en el mundo con mayor } \\
\text { número de especies vegetales, el biocomercio apenas empieza a } \\
\text { abrirse camino. En } 2006 \text {, con US\$307 millones exportados en } \\
\text { productos de biocomercio, Colombia tan sólo participa con el } \\
0,05 \% \text { del mercado mundial. }\end{array}$ & $\begin{array}{l}\text { Con la diversidad de ecología de Colombia en el } \\
\text { mundo su participación es muy baja, pero también } \\
\text { es importante reconocer que empieza hacer parte } \\
\text { de esta nueva generación. }\end{array}$ \\
\hline $\begin{array}{l}\text { (Vanguardia, 2017) } \\
\text { Los nuevos } \\
\text { comportamientos del } \\
\text { consumidor }\end{array}$ & $\begin{array}{l}\text { El comportamiento del consumidor en Colombia y en el mundo } \\
\text { está cambiando. Ahora se piensa más en el medio ambiente, en } \\
\text { comida saludable y en ahorrar recursos naturales, lo que para } \\
\text { algunos sectores se denomina "consumo responsable". }\end{array}$ & $\begin{array}{l}\text { Es alentador que el comportamiento del } \\
\text { consumidor en Colombia y el mundo entro esté } \\
\text { visualizado a cuidar la salud y el medio ambiente. }\end{array}$ \\
\hline $\begin{array}{l}\text { (La Opinion, 2017) } \\
\text { Más apoyo a negocios } \\
\text { verdes en norte de } \\
\text { Santander }\end{array}$ & $\begin{array}{l}\text { Con solo } 81 \text { empresas con potencial para transformar su } \\
\text { productividad hacia los negocios verdes, el departamento aún } \\
\text { tiene cabida para ampliar estas propuestas de las cuales también } \\
\text { se generan beneficios económicos derivados de actividades } \\
\text { amigables con el ambiente. }\end{array}$ & $\begin{array}{l}\text { A pesar de la inclusión de Norte de Santander en } \\
\text { particular en el mercadeo verde, aún le quedan } \\
\text { proyectos por incluir en esta nueva experiencia, } \\
\text { que generen ayuda a la conservación del medio } \\
\text { ambiente. }\end{array}$ \\
\hline $\begin{array}{l}\text { (Universidad de } \\
\text { Santander, 2016) } \\
\text { verde }\end{array}$ & $\begin{array}{l}\text { Los problemas ambientales pasaron de ser tratados sin } \\
\text { importancia hasta volverse una prioridad, por eso la Universidad } \\
\text { de Santander sede Cúcuta, comprometida en la formación de } \\
\text { profesionales de calidad, competentes y además ambientalmente } \\
\text { responsables, establece UDES VERDE; una estrategia que } \\
\text { fortalecerá y permitirá el cumplimiento de la política } \\
\text { institucional ambiental de la universidad. }\end{array}$ & $\begin{array}{l}\text { A causa del interés de toda la ciudad en estar } \\
\text { actualizado con las medidas que brinden una } \\
\text { amistad con el medio ambiente, la universidad de } \\
\text { Santander se ha unido comprometiéndose con la } \\
\text { formación de los profesionales en calidad, } \\
\text { competentes y ambientalmente responsables. }\end{array}$ \\
\hline
\end{tabular}

\section{Resultados y análisis}

El último objetivo de la investigación consistió en proponer una estrategia de comunicación de mercadotecnia a empresas cucuteñas. La finalidad de la estrategia fue el concientizar sobre el uso y venta de productos y servicios ecológicos en el segmento meta; por tanto, se obtuvo apoyo de las herramientas de comunicación de mercadotecnia como son: publicidad, relaciones públicas y mercadotecnia directa.

Así mismo en el punto se propone básicamente lo siguiente:

- Exposiciones en las universidades del mercado meta bajo el concepto del evento.

- Invitar a marcas locales y nacionales para que participen en el evento a fin de exponer las ventajas de los productos y servicios ecológicos en comparación con los tradicionales.

- Brindar información acerca de cómo ser más verde a las empresas contando con foros en donde lideres den su opinión, abordando temas como: reciclaje, separación de basura, ahorro de energía y ventajas de los productos ecológicos no solo para las organizaciones sino para el medio ambiente en general.

- Se propone que la mayor parte del material a utilizar sea ecológico.

- Se sugiere manejar un concepto verde.

- Repartir productos promocionales.

- Se recomienda que la organización, según sea el caso de la organización tenga presente la importancia de la comunicación de mercadotecnia para auxiliarse en ella y conseguir el posicionamiento de mensaje ecológico eficazmente. 
- Se propone que mediante la estrategia de comunicación propuesta se logre transmitir que los productos y servicios ecológicos no solo buscan crear una ventaja competitiva sino también buscan el apoyo hacia el cuidado del medio ambiente.

- Crear foros y grupos con información acerca del marketing verde en redes sociales.

- Hacer talleres con las empresas de Cúcuta, para brindar información sobre el mercadeo verde.

\section{Conclusiones}

En este trabajo se pudo observar que se ha aportado evidencia teórica y empírica en relación a los instrumentos del marketing y la decisión de compra ecológica. Así mismo se respondió a cada uno de los objetivos específicos del estudio, Además, se habla de los beneficios y la importancia que implica practicar el marketing ecológico como estrategia.

Esta investigación también nos relata que las miradas están puestas en las empresas sustentables que se preocupan por mantener un equilibrio entre la sociedad, la economía $y$ el medio ambiente y que al ofrecer al mercado productos y servicios sostenibles estas organizaciones se estarían ayudando a sí mismas en publicidad y reconocimiento, al aumentar la cantidad de consumidores que buscan productos que no afecten al medio ambiente y a la vez sea de buena calidad también aumenta la competencia en el mercado y de la mano estrategias que cubran las necesidades de los clientes y las necesidades de la empresa.

\section{Referencias}

Acosta, F. (1985). El Marketing Social. Recuperado de http://catarina.udlap.mx/u_dl_a/tales/ documentos/lco/soto_a_ig/capitulol.pdf

Catarina. (2008). Acervos Digitales. Recuperado de http://catarina.udlap.mx

Chamorro. (2001). "El Marketing Ecológico".

Dinero. (2017). Dinero. Recuperado de http:// www.dinero.com/edicion-impresa/especialcomercial/articulo/el-negocio-del-futuro/50994

Hartmann . (2004). Universidad de Valencia. Recuperado de https://www.uv.es

Kerlinger. (2002). Investigación del comportamiento.

La Opinion. (2017). Más apoyo a negocios verdes en Norte de Santander. La Opinión. Recuperado de https://www.laopinion.com.co/ zona-verde/mas-apoyo-negocios-verdes-ennorte-de-santander-139263

Landazal, J. (2009). El progreso a través de la revolución industrial. Obtenido de http://www. eumed.net/cursecon/libreria/2004/hjmc/1b. htm

Sampieri, H. (2014). Metodologia de la investigacion. McGraw-Hill Education.

Sanchez, N. (2000). Marketing ambiental. Obtenido de http://www.esacademic.com/dic. nsf/eswiki/1369831/Marketing_ambiental

Terrón. (2007). ftp.repec. Obtenido de ftp://ftp. repec.org

Universidad de Santander . (2016).

Vanguardia. (2017). Vanguardia. Obtenido de http://www.vanguardia.com/ economia/negocios/398379-los-nuevoscomportamientos-del-consumidor 